\title{
Possible Exotic phases in the One-Dimensional Extended Hubbard Model
}

\author{
R. Torsten Clay, Anders W. Sandvik, and David K. Campbell \\ Department of Physics, University of Illinois at Urbana-Champaign, 1110 W. Green Street, Urbana, Illinois 61801
}

(April 18, 2018)

\begin{abstract}
We investigate numerically the ground state phase diagram of the one-dimensional extended Hubbard model, including an on-site interaction $U$ and a nearest-neighbor interaction $V$. We focus on the ground state phases of the model in the $V \gg U$ region, where previous studies have suggested the possibility of dominant superconducting pairing fluctuations before the system phase separates at a critical value $V=V_{\mathrm{PS}}$. Using quantum Monte Carlo methods on lattices much larger than in previous Lanczös diagonalization studies, we determine the boundary of phase separation, the Luttinger Liquid correlation exponent $K_{\rho}$, and other correlation functions in this region. We find that phase separation occurs for $V$ significantly smaller than previously reported. In addition, for negative $U$, we find that a uniform state re-enters from phase separation as the electron density is increased towards half filling. For $V<V_{\mathrm{PS}}$, our results show that superconducting fluctuations are not dominant. The system behaves asymptotically as a Luttinger Liquid with $K_{\rho}<1$, but we also find strong low-energy (but gapped) charge-density fluctuations at a momentum not expected for a standard Luttinger Liquid.
\end{abstract}

PACS numbers: 71.10, 74.20.Mn

\section{INTRODUCTION}

One-dimensional (1D) Hubbard models have been used to model many quasi-1D systems, including conducting polymers such as polyacetylene, 0 and organic charge-transfer materials such as TTF-TCNQ or (TMTSF) ${ }_{2} \mathrm{PF}_{6}$. 2 In the simplest form, with only an onsite interaction $U$, the 1D Hubbard model has been solved exactly using the Bethe Ansatz. ${ }^{3}$ However, longer range interactions are needed to describe many effects observed in real materials, e.g., the excitons found in conducting polymers. 6 The conventional extended Hubbard model (EHM), which in addition to $U$ includes a nearestneighbor interaction of strength $V$, is the simplest extension that takes into account some of the longer-range interaction effects. The EHM Hamiltonian is

$$
\begin{aligned}
H= & -t \sum_{i \sigma}\left(c_{i+1 \sigma}^{\dagger} c_{i \sigma}+c_{i \sigma}^{\dagger} c_{i+1 \sigma}\right)+U \sum_{i} n_{i \uparrow} n_{i \downarrow} \\
& +V \sum_{i} n_{i} n_{i+1},
\end{aligned}
$$

where $c_{i \sigma}^{\dagger}$ creates an electron of spin $\sigma$ on site $i$, and $n_{i \sigma}=$ $c_{i \sigma}^{\dagger} c_{i \sigma}\left(n_{i}=n_{i \uparrow}+n_{i \downarrow}\right)$ are electron number operators. An implicit parameter is the filling factor $n=N_{e} / N$, where $N_{e}$ and $N$ are respectively the number of electrons and lattice sites. We will henceforth give energies in units of the hopping $t$.

As one of the basic many-body Hamiltonians in 1D, the EHM has been the subject of a large number of studies. 0 Nonetheless, as we shall discuss below, there remain important open questions related to the phase diagram at intermediate and strong coupling, where both analytical and numerical methods are difficult to apply reliably. One of the principal reasons for the existence of these open questions is the variety of potential broken symmetry fluctuations that can occur in the EHM.
As the parameters $U, V$, and $n$ are varied, several different types of correlations dominate the ground state, including spin or charge density wave (SDW/CDW), and singlet or triplet superconducting (SC) fluctuations. Of course, in a strictly $1 \mathrm{D}$ model, long-range order that breaks a continuous symmetry (i.e., SC or SDW) is not possible; however long-range CDW order can occur at zero temperature. Further, in some parameter regions, the EHM is unstable towards phase separation (PS), with the nature of the coexisting phases depending on the parameters.

For small values of the interaction parameters, the lowenergy excitations of the EHM can be mapped directly to a "Luttinger liquid" (LL),11 the unifying framework for 1D interacting fermion systems obtained from weakcoupling renormalization group studies, bosonization, and conformal field theory. The general ( $q$-component) LL contains $q$ gapless degrees of freedom, and the forms of the correlation functions depend on only two parameters for each gapless mode $\alpha$ : A renormalized velocity $v_{\alpha}$ and an interaction parameter $K_{\alpha}$ Epr instance, the standard Tomonaga-Luttinger mode 12.13 has two gapless degrees of freedom, charge and spin, and is thus a twocomponent LL with interaction parameters $K_{\rho}$ and $K_{\sigma}$; a similar one-dimessional exactly solvable model, due to Luther and Emery, 14 has gapless charge excitations, but a spin gap, and thus behaves as a Luttinger liquid only in the charge sector. The identification of a given model as a Luttinger liquid enables (in principle) a straightforward numerical investigation of the ground state phases of the model. In the case of the integrable standard Hubbard model, the Bethe Ansatz equations have been used this way to calculate the ground state parameters and $K_{p}$ for all values of the repulsion $U$ and the band fillings. 15 For more general models, away from weak coupling, there is unfortunately no reliable analytic method to determine the parameters, although they may in principle be calcu- 
lated numerically. Assuming the model remains a LL also away from the weak-coupling regime, numerical methods can be used to calculate the exponents $K_{\alpha}$, which will then give the asymptotic form of the correlation functions. Importantly, for such calculations to be meaningful, finite-size and other systematic errors must be carefully analyzed, and in particular, one must ascertain that the model is not phase separated in the thermodynamic limit. As we demonstrate below, calculating Luttinger liquid parameters from finite-size systems can sometimes be highly problematic, particularly close to phase separation.

The existence of several distinct regions and typefs phase separation in the phase diagram of the FHM $6.09,0$ and other 1D strongly-correlated models $16-19$ is wellestablished. While phase separation typically occurs outside the parameter regions thought to be relevant for modeling real materials, for purposes of understanding the behavior of any given theory it is imperative that all phase-separated regions be identified and, if possible, understood prior to carrying out other studies, such as determinations of Luttinger liquid correlation exponents. In small systems, the signals for phase separation can be unclear or ambiguous. For instance, the correlation functions calculated for small systems can be misleading in phase-separated regions, since the boundary between the two phases can be large compared to the system size. This leads to a mixing of correlations from the two different phases, which may have quite different properties (for example, spin-gapped and non-spin-gapped). Nonetheless, in a small system, these correlations may not show signs of phase separation until well inside the phase-separated region. Sometimes, it may then appear that the exponent $K_{\rho}>1$ (which would signal dominant superconducting correlations), when in fact the system is phase separated in the thermodynamic limit and is thus not a uniform Luttinger liquid. Accordingly, one of our principal goals in this study has been to understand thoroughly the phase-separated regions before calculating Luttinger liquid exponents or other correlation functions.

Recently, the extended Hubbard model in the region of parameters $V \gg U \sim t$ has attracted considerable interest because of the possibility of a novel superconducting ground state away from half-filing $(n<1)$.6 Although the interactions appear to be purely repulsive when $V>U>0$ (we also consider $V \gg U$ with $U<0$ ), the gradient of the potential is positive, and there is an attractive force between electrons. Pairs might then form and could in principle lead to a ground state with dominant superconducting fluctuations. Based on Lanczös exact diagonalization (ED) results at quarterfilling $(n=1 / 2)$, it was argued that superconducting fluctuations indeed dominate for a substantial range of $V$ values, befgre the system phase separates at a very large $V=V_{\mathrm{PS}}$.6 Another ED study found similar behavior at filling $n=2 / 3$, but no attempt was made to determine whether the system is phase separated at this filling.
These previous studies also addressed the question of the location of the spin gap boundary. Determining the location of the spin-gapped Luther-Emery phase is important to studying the possibility of superconductivity in this region, as the presence of a spin gap would be consistent with the expected dominant singlet pairing correlations when $V$ is large and positive. Superconducting correlations involving triplet pairs have also been proposed as a scenario to explain ED results Triplet superconductinf correlations are dominant in some regions with $V<0$ (10), but explaining their origin for $V \gg|U|$ is problematic.

In this paper, we investigate the related questions of novel superconducting fluctuations, calculations of $K_{\rho}$, and the boundaries of phase separation and spin-gapped regions using quantum Monte Carlo (QMC) simulations of relatively large systems (up to 128 sites). We focus on the ill-understood parameter region $V \gg|U|$ for a wide range of fillings. Our results show that phase separation extepds to much lower values of $V$ than previously reported.6. We also find that for negative $U$ the highdensity phase is not the naively expected one with doubly occupied sites separated by one site (corresponding to half filling in the high-density phase) but is at a lower density. A uniform state (which has strong CDW fluctuations) re-stabilizes as half filling is approached. In most of the parameter space we can conclude that $K_{\rho}<1$ for $V<V_{\mathrm{PS}}$. In some cases, our results are on the borderline $K_{\rho} \gtrsim 1$ at our calculated phase separation boundary, but in no case do we find a definite $K_{\rho}>1$ before phase separation. We therefore believe that superconducting correlations never dominate in the $V \gg|U|$ region. Instead, for a range of parameters, we find strong chargedensity fluctuations at a momentum $2 k_{\mathrm{F}}<q<4 k_{\mathrm{F}}$, which are not expected in a standard Luttinger liquid. Our analysis of the temperature and size dependence of these fluctuations shows that they do not correspond to gapless excitations, and hence the model remains a Luttinger liquid in the asymptotic low-energy sector. Nevertheless, these strong anomalous fluctuations demonstrate the appearance of non-Luttinger liquid features in the excitation spectrum.

To present our results, we begin in Sec. II by discussing briefly the numerical methods we have used to study the extended Hubbard model. We point out advantages and disadvantages of several different QMC methods and stress the necessity of comparing and contrasting their predictions to obtain definitive conclusions. In Sec. III, we discuss methods to detect phase separation in numerical data, emphasizing a number of often overlooked subtleties, and present our results for the phase separation boundaries of the extended Hubbard model. We address the determination of Luttinger liquid exponents and other correlation functions in Sec. IV. Sec. V concludes with a summary of our understanding of the "exotic" phases of the extended Hubbard model in the region $V \gg|U|$. 


\section{NUMERICAL METHODS}

Using the Luttinger liquid formalism, extracting the dominant correlations of $1 \mathrm{D}$ electron systems is easy in principle. Lanczös exact diagonalization of small systems can give results for certain observables (e.g., velocities and stiffness constants) that can be used to determine the asymptotic form of the correlations functions which are believed to be less affected by finite-size effects than the correlation functions themselves. This has led to an upsurge of ED studies of various 1D models, including many focusing on possibilities of dominant supercpudycting fluctuations close to phase separation.6 6 $16.17,20,22$ However, as we argue below, the finite-size effects may in fact be unexpectedly large in regions near phase separation. It is therefore important to confirm ED results using methods that can treat larger system sizes. For this purpose, we have used three different QMC methods. Here we summarize briefly the basic ideas of these techniques, referring readers to the literature for additional details. Our main purpose is to make some observations concerning the advantages and disadvantages of the different QMC methods in studies of the EHM in the difficult parameter regime $V \gg|U|$. We believe that most of this discussion will apply to the region of very strong interactions in other models as well.

The first method is based on the "stochastifseries expansion" (SSE) of the density matrix $e^{-\beta H} 23,24$ This is a generalization of Handscomb's method,25 applicable for a much larger class of lattice Hamiltonians. There are no approximations such as Trotter discretization of imaginary time, but in order to obtain ground state results one has to ensure that a large enough inverse temperature $\beta$ is used. To check for finite-temperature effects, we have carried out calculations for several values of $\beta$. In general, we find that $\beta \sim 2 N-4 N$ is sufficient to give accurate values for the ground state parameters for $N$ up to 128 .

The second technique is a recently developed variant of the continuou-time worldline algorithm proposed by Prokofev et al.e2 Our version of this method20 uses an updating scheme adapted from the SSE algorithm the two methods are, in fact, closely related to each other 27). The algorithm is based on the finite-temperature perturbation expansion in the interaction representation, around the atomic-limit system with no kinetic energy term. This expansion converges for a finite system at finite $\beta$. The terms (which are paths in continuous imaginary time) can be stochastically sampled in much the same way as is done in the SSE method. We will refer to this QMC technique as the interaction representation (IR) method. It is also free from systematic errors.

Results of SSE and IR simulations are in general in complete agreement with each other. Both methods operate in the real-space occupation number basis and hence suffer from well known "sticking problems" (inability of the local Monte Carlo updates to evolve the real space configurations through states with high potential energy) when the interactions are very strong. For the EHM, $V \approx 10$ seems to be the highest accessible $V$ in the interesting filling regions ( $U$ represents a lesser problem, since we consider $V \gg U)$. We have found that the autocorrelation times for spin and density correlation functions at strong interactions are shorter for the IR method, in particular close to half filling. All results presented here for correlation functions and susceptibilities were therefore obtained with that method. However, for the ground state energy (i.e., the internal energy at sufficiently high $\beta$ ), the SSE method gives results with statistical errors approximately an order of magnitude smaller than the IR method (in cases where the sticking problems do not make simulations practically impossible). This result probably arises because the total energy estimator of the SSE method is directly related to the stochastically averaged order of the terms of the expansion of $e^{-\beta H}$. In the IR method (and other worldline methods), the energy estimator consists of separate diagonal and off-diagonal parts, i.e., the potential and kinetic energies are not treated on an equal footing (in particular, the diagonal part is typically not spinrotationally invariant). We note that the SSE method also has proven superior in energy calculations for spin models, such as the two-dimensional Heisenberg model,28 for which otherwise very efficient loop algorithms 29 have not given nearly as accurate results.

The third QMC method we employ is the recently developed Constrained Path Monte Carlo (CPMC) algorithm. This is a Slater-determinant based projector method that handles the interactions via a Trotter decomposition and a Hubbard-Stratonovich transformation, leading to auxiliary fields that are sampled.30,31 A constraining wavefunction is used to prevent the fermion sign problem. In 1D, the constraint becomes exact (i.e., there are no sign problems). There is a small systematic error originating from the Trotter decomposition, which can be made arbitrarily small by decreasing the "imaginary time slice" width. In our comparisons with SSE and IR results, CPMC yielded similar results, except at very strong interactions where matrix conditioning problems become overwhelming and make the method very hard to use (much before the sticking problems become problematic in SSE and IR simulations). One advantage of CPMC over the SSE and IR methods is that in CPMC any equal-time correlation function may be computed since the Green's function is directly accessable in CPMC. To obtain accurate energies from CPMC it was necessary to compute the energy for more than one $\Delta \tau$ value and scale the results to $\Delta \tau \rightarrow 0$. Our implementation of CPMC used a uniform free-electron wavefunction for the constraint, the initial wavefunction, and the importance sampling wavefunction. While the choice of the importance sampling wavefunction should not affect the final results, if the overall symmetry is incorrect the method becomes very inefficient. In phase separated regions, we found that using the uniform impor- 
tance wavefunction resulted in larger statistical errors; unfortunately there is no easy way to construct a phaseseparated trial function in this case. This is another example of the great care that must be taken in chosing an appropriate trial function in projector methods32. In Table I we compare energies calculated using the SSE and CPMC methods for 32 site systems with $U=1$ and $V=8$. For the rest the results in this paper, we have used the SSE and IR methods exclusively, using SSE for energies when possible and IR for structure factors and susceptibilities.

\begin{tabular}{|l||l|l|l|}
\hline \hline $\mathrm{n}$ & IR & SSE & CPMC \\
\hline 0.3125 & $-0.461(2)$ & $-0.4644(2)$ & $-0.4656(2)$ \\
0.3750 & $-0.451(2)$ & $-0.4484(2)$ & $-0.4506(2)$ \\
0.4375 & $-0.382(3)$ & $-0.3825(1)$ & $-0.3829(2)$ \\
0.5000 & $-0.293(3)$ & $-0.3000(2)$ & $-0.3002(3)$ \\
0.5625 & $-0.211(3)$ & $-0.2105(2)$ & $-0.2108(4)$ \\
0.6250 & $-0.113(3)$ & $-0.1179(2)$ & $-0.1168(6)$ \\
0.6875 & $-0.025(4)$ & $-0.0261(2)$ & $-0.0200(6)$ \\
0.7500 & $-0.070(3)$ & $0.0629(3)$ & $0.072(1)$ \\
0.8125 & $0.150(2)$ & $0.1510(2)$ & $0.1640(7)$ \\
0.8750 & $0.239(2)$ & $0.2406(3)$ & $0.245(1)$ \\
\hline
\end{tabular}

Table I: Comparison of QMC energies per site for $V=8$ and $U=1$. Statistical errors in last digit are indicated in parentheses. The CPMC results used a free-electron trial function and were scaled for $\Delta \tau \rightarrow 0$ from $\Delta \tau=0.1$ and $\Delta \tau=0.05$.

\section{PHASE SEPARATION}

\section{A. Phase Separation and Superconductivity}

We have already noted that an obvious motivation for mapping carefully the regions phase separation in the extended Hubbard model is that in a variety of stronglycorrelated models, superconductivity has been found, or argued to be present, in close proximity to phase separation boundary. Such proximity is intuitively reasonable, since both superconductivity and phase separation result from effectively attractive interactions. The case of the EHM for $V<0$ provides an illustration. In this region the model phase separates into a low-density phase and a high-density phase; the high-density phase consists either of adjacent doubly occupied sites or of adjacent single electrons, depending on the value of $U .9,10$ Near these phase-separated regions at negative $V$ are well-established regions of singlet and triplet superconducting fluctuations.

However, the possible proximity of superconductivity to phase separation is also problematic, for if one applies the Luttinger liquid formalism and numerical techniques to calculate $K_{\rho}$ for relatively small systems in a region that is in fact phase separated in the thermodynamic limit, one will obtain incorrect results. Hence, before a mapping to Luttinger liquid parameters can be used, the phase separation boundaries of a model should be accurately determined. To quantify this point, we here note that in one recent study of the EHM at quarter filling the phase separation boundary for $V \gg|U|$ was determined using a cluster Gutzwiller approximation to find the vanishing of the inverse compressibility. The smallest $V$ (for any of the values of $U$ studied) for which phase separation was found was $V \sim 14$ (for $U \sim-2.5$ ) An exact diagonalization study gave comparable results.6 Our QMC studies of larger systems reveal that phase separation occurs already at $V \approx 8$ at quarter filling, in close proximity to where these previous studies indicated that dominant superconducting fluctuations first appear.

\section{B. Strong-Coupling calculations in the $V \rightarrow \infty$ limit}

An effective model, first derived by Penc and Mila (PM) conveniently captures the exact behavior of the EHM for $V \rightarrow \infty$. For infinite $V$, any existing pairs cannot be broken up (or moved), while single electrons cannot occupy neighboring sites and hence behave as spinless fermions. The effective model thus consists of immobile pairs and single spinless electrons. For parameter values where pairs and unpaired electrons coexist, the minimum energy corresponds to having the pairs and the spinless fermions separated into two distinct regions, so as to minimize the kinetic energy of the spinless fermions by allowing them to move in the largest possible region. The pairs are separated by one lattice spacing. The energy for a given number of pairs and single electrons can then readily be calculated analytically in the thermodynamic limit as the sum of $U$ times the number of pairs plus the energy of a spinless fermion chain:

$$
E / N=\frac{m n U}{2}-t \frac{2}{\pi}(1-n) \sin \left[\pi \frac{n}{1-n}(1-m)\right] .
$$

Here $m$ is the fraction of fermions forming pairs in the ground state, $E / N$ is the energy per lattice site. The energy can be minimized with respect to $m$ to determine the ground state. For $U<-4$, one can show that all electrons are paired for all fillings, giving a spin gap and the boundary of the Luther-Emery region for $V \rightarrow \infty$. For $U>-4$, the ground state contains only unpaired electrons for fillings less than a critical filling, above which the pair phase starts to grow. At half filling, the system contains only pairs. Equation (2) thus provides an exactly solvable model exhibiting phase separation.

While PM focused exclusively on the quarter-filled case, their energy expression (2) can be used to obtain the phase-separated region for all fillings $n$. If the system is phase separated with the high- and low-density phases at densities $n_{\mathrm{HD}}$ and $n_{\mathrm{LD}}$, respectively, then phase separation occurs for the total (average) densities $n$ in the range $n_{\mathrm{LD}}<n<n_{\mathrm{HD}}$. In the thermodynamic limit the ground state energy must be linear as a function of $n$ in this regime, reflecting the fact that adding particles 
to the system only changes the relative sizes of the two phases in a system with fixed particle number (canonical ensemble).

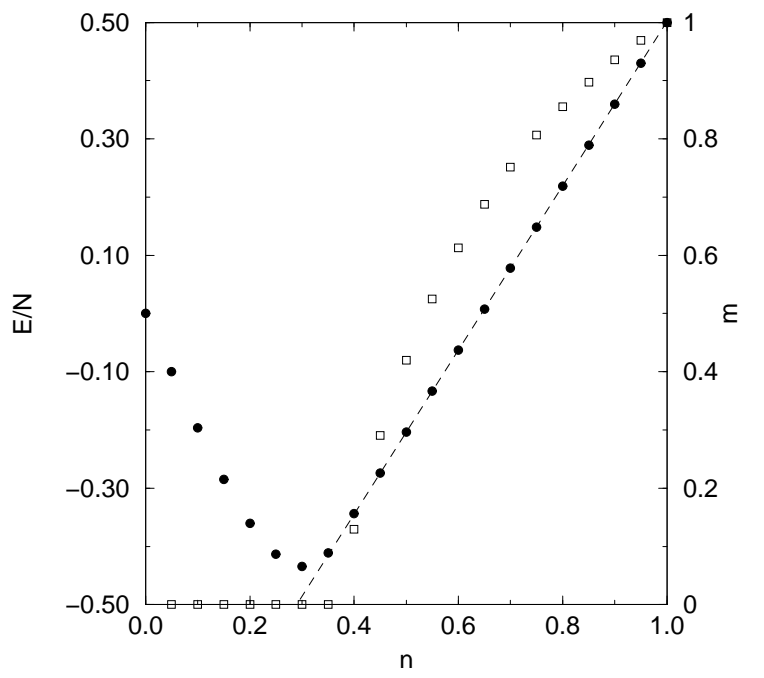

FIG. 1. The exact energy per site for for $V \rightarrow \infty$ and $U=1$ (solid circles, left axis) calculated using Eq. (2). The dependence on $n$ is completely linear for $n>0.37$, as indicated by the dashed line, reflecting phase separation. The open squares (right axis) denote the fraction $m$ of fermions forming pairs in Eq. (2).

Figure 11 shows the energy per site versus filling in the $V \rightarrow \infty$ limit. At low fillings the system contains only unpaired electrons. Above a critical filling $n_{\mathrm{LD}}$, the energy becomes linear, reflecting phase separation as pairs are formed. The linear behavior persists all the way up to half filling ( $\left.n=1=n_{\mathrm{HD}}\right)$, because the high density phase consists of pairs occupying every other site. The phase separation density can be easily calculated as a function of $U$, resulting in the phase diagram shown in Figure 2.

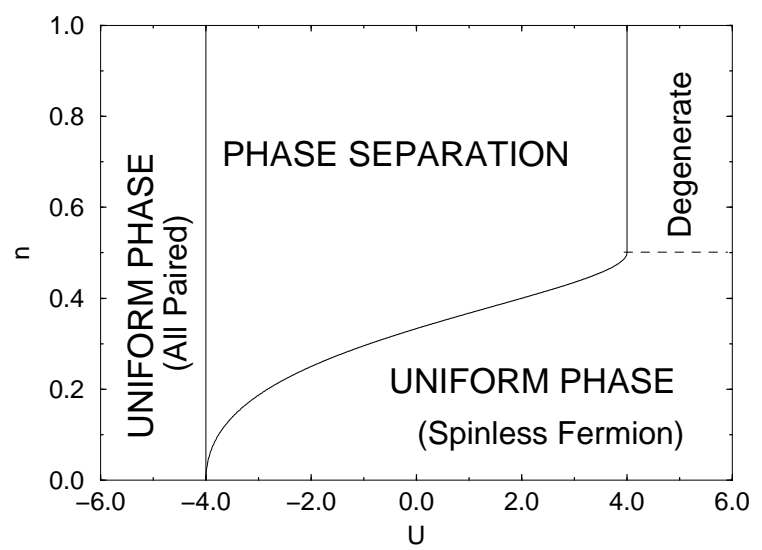

FIG. 2. The region of phase separation (PS) (extending from $U=-4$ to $U=+4$ ) for infinite $V$. The other phases are discussed in the text.

For $U>4$, the phase-separated and uniform states be- come degenerate at $V \rightarrow \infty$, which we label as non-phase separated because we expect any finite $V$ will break the degeneracy and the resulting state will not be phase separated. Mila and Zotos have shown that this state in fact is uniform and insulating.6 For finite $V$, we expect a smaller phase-separated region, as pairs will be able to break up with a finite amount of energy. Thus we are able to confine our numerical investigation of phase separation to the range $-4<U<4$.

\section{Numerical Calculation of the PS Boundary}

Numerically, for finite $V$, the phase separation boundary can be determined using the energy as a function of the number of particles in the system, as discussed above. However, a completely linear behavior will not be observed in a finite system, because the boundaries between the high- and low-density phases then occupy a significant fraction of the lattice and raise the energy per site by an amount that depends on the size of the boundary regions (we use periodic systems and hence have two boundaries). This will cause the energy as a function of $n$ to be concave, which is not possible in the thermodynamic limit. A line can be drawn which is tangent to the $E(n)$ curve at two points, which then constitute estimates of the fillings $n_{\mathrm{LD}}$ and $n_{\mathrm{HD}}$ of the high- and low-density phases. In the absence of phase boundaries (which become irrelevant to the energy per site in the thermodynamic limit), the line corresponds to $E / N$ of a system separated into regions of densities $n_{\mathrm{LD}}$ and $n_{\mathrm{HD}}$. For small system sizes, this "Maxwell construction" can be expected to be more accurate than signals of phase separation based on, e.g., probability distributions of particle densities, since the ground state energy typically exhibits only small finite-size effects in cases where the thermodynamic limit state is uniform. It was previously assumed that $n_{\mathrm{HD}}=1$ also for finite $V$ As we will see, in fact $n_{\mathrm{HD}}<1$ in some parameter regions. In any case, we will refer to $n_{\mathrm{LD}}$ as the phase separation density.

As a complementary method of determining the phase separation boundary, as well as to help in characterizing the phases, we have also used a criterion based on the static charge structure factor $S_{\rho}(q)$ :

$$
S_{\rho}(q)=\frac{1}{N} \sum_{j l} e^{i q(j-l)}\left\langle n_{j} n_{l}-n^{2}\right\rangle .
$$

Continuity at $q=0$ requires $S_{\rho}(q \rightarrow 0)=0$ in a uniform system. In a phase-separated periodic system of size $N$ in the canonical ensemble, $S_{\rho}(q)$ will have a maximum at the shortest non-zero wave-number $q=q_{1}=2 \pi / N$, with a divergence as $N \rightarrow \infty$. In small systems close to the boundary of phase separation, this signal is, however, ambiguous, since there will be a range of parameters for which it is not possible to determine accurately the $S(q \rightarrow 0)$ behavior based on the behavior for $q \geq q_{1}$. We shall see examples of this in later sections. 
Returning to the Maxwell construction, we note that for it to be reliable one must be certain that the energy can be computed accurately. Although there are no $a$ priori approximations in two of the QMC methods we use, near half filling at large $V$, we have noticed that the QMC simulations may converge poorly, apparently becoming stuck in meta-stable states. This occurs because the ground state near half filling contains a significant fraction of on-site pairs that require considerable energy to break up or move, making it difficult for the simulation to explore the full phase space of the model. The simulations are particularly hard when the ground state is phase separated. The system can then separate into several alternating domains of high-and low-density phases, instead of just one of each. The resulting large statistical errors in the energy close to half filling cause problems in the determination of the tangent line and thus the point of phase separation. Fortunately, exactly at $n=1$, the system is always uniform and the ground state energy can be calculated very accurately using exact diagonalization, since the finite size effects are very small at this filling (much smaller than for $n<1$ ). This can be easily understood from perturbation theory around the static $(t=0)$ ground state, which is non-degenerate at $n=1$ (and only there) and consists of alternating doubly occupied and empty sites (i.e., a period-two CDW). A simple second-order perturbation calculation gives an energy per site of

$$
E / N=\frac{U}{2}-\frac{2 t^{2}}{3 V-U}+\ldots
$$

This thermodynamic expression compares remarkably well with exact diagonalizations on small systems; for example with $U=1$ and $V=10$, it gives $E / N=0.431034$, while the exact results for $N=6$ and $N=12$ are $E / N=0.431092$ and $E / N=0.431096$. Unfortunately, the smallness of the finite-size effects holds only exactly at half filling (since the particles are no longer localized once the system is doped away from half filling).

For $n \lesssim 0.7$, the QMC simulations converge well even for phase-separated ground states (for $V / t \leq 10$ ), and we have been able to calculate $E / N$ to within absolute statistical errors of $\sim 10^{-4}-10^{-3}$ using the SSE method (energies for small systems agree with ED results). Figure 3 shows our Maxwell construction for $N=64, U=1$ and $V=8$. Also shown is a plot of $\Delta E$, the difference between the $E / N$ values and the best fit tangent line. Using this method, we find that the system in this case phase separates at $n$ slightly above quarter filling. Data for $N=32$ give the same result, indicating that this is indeed close to the phase separation point in the thermodynamic limit. Thus, for a quarter filled system, we can conclude that $V_{\mathrm{PS}} \approx 8.0$ for $U=1$ ( since for $V=8$, phase separation occurs at $n$ only slightly above $1 / 2$, and the critical filling decreases with increasing $V$ ). Previous studies at this filling 6 , found a $V_{\mathrm{PS}} \approx 14-18$ at $U=1$. Our phase separation boundary is hence at significantly lower $V$ than reported and is very close to the $K_{\rho}=1$ curve obtained using exact diagonalization 6 , This is a clear indication of possible problems with the prediction of a novel superconducting region for $V \gg|U|$.

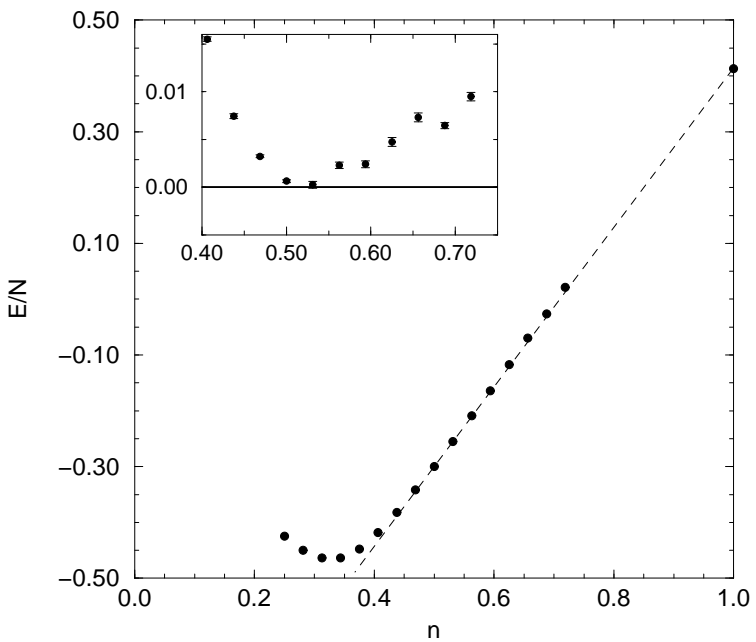

FIG. 3. Maxwell construction for $V=8$ and $U=1$. The solid circles are energies calculated for $N=64$. The dashed line goes through the $n=1$ energy, and is tangent to the energy curve at the phase separation filling (density of the low-density phase) $n_{\mathrm{LD}} \approx 0.53$. The inset shows the difference between the data points and the tangent line.

A definite disadvantage of using the Maxwell construction method for determining phase separation boundaries is that the need to calculate very precise energies causes this approach to be extremely time consuming. Accordingly, as already noted, we employed also a second method of detecting phase separation, which relies on the behavior of the static charge structure factor defined in Eqn. (3) for $q \rightarrow 0$. Figure 4 shows $S_{\rho}(q)$ for several densities at $U=-1,0,1$, and $V=8$. For $U=1$ we obtain phase separation at a density $n \approx 0.59$ from $S_{\rho}(q)$, compared to $n \approx 0.53$ from the Maxwell construction. Note, however, that at $U=1$ and $n=9 / 16, S_{\rho}(q)$ shows no phase separation, whereas the Maxwell construction implies the system is already phase separated. $S_{\rho}(q)$ gives a slightly smaller phase-separated region than the Maxwell construction because a clear signal of an increase as $q \rightarrow q_{1}$ for a relatively small lattice requires that the system already be well inside the region of phase separation.

Examining the region of phase separation for $U \leq 0$ reveals several interesting properties: whereas at $U=1$ the system stays phase separated for all densities $n<1$ above some critical density $n_{\mathrm{LD}}$, for $U=0$ we see clear signs that phase separation disappears at high densities. This can be seen in the structure factors in Figure 4, which go smoothly to zero for $U=-1,0$ and $n>0.9$. The Maxwell construction is also consistent with phase separation to a state with a high-density phase at filling less than $n=1$, although the relatively large statistical errors in the energies close to half filling make it difficult 
to obtain an independent estimate of $n_{\mathrm{HD}}$. However, investigating the charge structure factor for several system sizes, it is clear that the uniform phase for $n \lesssim 1$ is stable. The peak position of the charge susceptibility is also consistent with a high-density phase different from the $U=1$ case (see next section). We conclude that there is a "re-entrant" uniform state for $U \sim 0$. In addition, from simulations carried out at larger $V(V=10)$, we see the phase-separated region moving further into the $U<0$ side of the phase diagram and the re-entrant uniform state remains. As we will discuss in the next Section, the re-entrant state has strong $2 k_{\mathrm{F}} \mathrm{CDW}$ fluctuations.

We believe that the the re-entrance of the uniform state for $U \lesssim 0$, but not for $U>0$, can be qualitatively understood in the following way: When $V$ is large and $n \rightarrow 1$, pairs are formed to avoid the nearest-neighbor repulsions. For $U>0$, the pairs have positive energy.

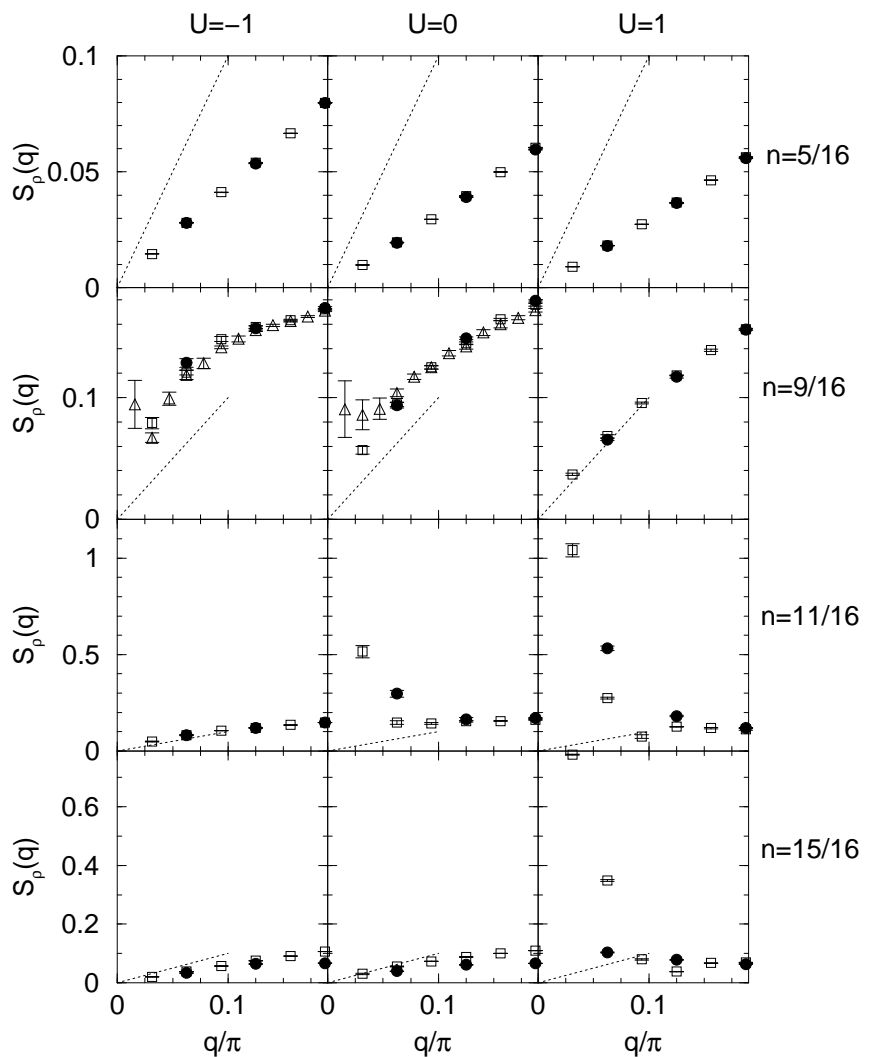

FIG. 4. The static charge structure factor $S_{\rho}(q)$ for small momenta $q / \pi$ at various fillings for $V=8$ and $U=-1,0,1$. Filled circles are for $N=32$, open squares for $N=64$ and triangles for $N=128$. As explained in Section IV, the straight lines all have slope $=\pi$, corresponding to the $q \rightarrow 0$ behavior if the LL exponent $K_{\rho}=1$.

Via phase separation, the total energy is minimized by balancing the potential energy of the pairs (which increases with the number of pairs) and the kinetic energy of a low-density phase with dominantly unpaired electrons. The pressure of the unpaired phase forces the distance between the pairs in the immobile high-density phase to be minimized to one lattice spacing (the kinetic energy of a pair is very small due to the $V$ barrier to hopping, and the motion of the pairs can be be neglected). The combined effects of avoiding the repulsive on-site $U$ and delocalization of mobile unpaired electrons always favor phase separation as $n \rightarrow 1$ if $V$ is large.

On the other hand, for $U<0$, the pairs have negative energy and maximizing their number is favorable. For $n$ close to 1 a uniform state with mobile pairs can then be stabilized if $V$ is not too large. However, as the density is lowered the number of negative-energy pairs decreases, and if $|U|$ is small a phase separated state can then again be lower in energy due to the lower kinetic energy of dominantly unpaired electrons in the low-density phase. Increasing $|U|(U<0)$, maximizing the number of mobile pairs becomes more favorable, and the size of the region of phase separation decreases, in agreement with our results.

For $U>1$ at $V=8$, we find that the phase separation boundary moves towards higher fillings, becoming hard to discern for $U \sim 4$, as expected from the infinite $V$ solution. For $U \lesssim-1$, based on 32-, 64- and limited 128-site data, phase separation does occur for $V=8$, but is much harder to detect than at $U=1$ and $U=0$. Increasing $V$ to 10 , we cannot obtain as accurate results as for $V=8$, but we do find clear signs that the phase separation region moves to lower values of $n$, as expected if there is a continuous evolution of the phase separation boundary to the infinite $V$ solution discussed in the previous section. We also find that phase separation does not occur at all for $V=4$, and for $V=6$ appears to be present only in a small region for $U>0$.

Finally, we stress the need to use more than one system size in applying the approach based on $S_{\rho}(q)$, as otherwise the analysis may give ambiguous or erroneous results for the phase separation boundary. For instance, based on the 32- and 64-site results shown in Fig. .4, there would appear to be no phase separation at $U=-1$ and the slope of $S_{\rho}(q)$ versus $q$ as $q \rightarrow 0$ exceeds $\pi$ for some fillings. This would imply (see next section) that $K_{\rho}>1$ and therefore dominant superconducting correlations in this region. However, based on data for larger $(N=128)$ lattices, it is likely that the system is phase separated at these fillings (see $n=9 / 16$ for both $U=-1$ and $U=0$ in Figure 1 ).

\section{CORRELATIONS AND FLUCTUATIONS}

\section{A. Methods to calculate LL parameters}

In this section we describe results of calculations for a variety of correlation functions of the ground state of the EHM in non-phase-separated regions using various different estimators for the Luttinger liquid correlation exponents $K_{\rho}$ and $K_{\sigma}$. For models (like the EHA) with spin-rotation symmetry, the exponent $K_{\sigma}=133$ except 
in the Luther-Emery phase, in which case only $K_{\rho}$ has meaning, since the system is spin-gapped and not a LL in the spin sector. For $K_{\sigma}=1$, the asymptotic equaltime correlation functions in a LL have simple power law forms governed by $K_{\rho}$ :

$$
\begin{aligned}
N_{\mathrm{SDW}, \mathrm{CDW}}(r) & \sim r^{-\left(1+K_{\rho}\right)} \cos \left(2 k_{F} r\right), \\
N_{\mathrm{SS}, \mathrm{TS}}(r) & \sim r^{-\left(1+1 / K_{\rho}\right)} .
\end{aligned}
$$

Hence, when $K_{\rho}<1$, SDW/CDW correlations dominate at large $r$, while for $K_{\rho}>1$, superconducting correlations dominate. At weak coupling, the renormalization group "g-ology" procedure can be used to determine the exponents $K_{\rho}$ and $K_{\sigma} .345$ Away from weak coupling, one must use the Luttinger liquid equations that relate $K_{\rho}$ to other physical observables of the model. In our present notation, the relations we use are 15

$$
\begin{aligned}
& K_{\rho}=D_{\rho} / 2 v_{\rho}, \\
& K_{\rho}=\pi v_{\rho} \kappa / 2, \\
& K_{\rho}=\left(\pi \kappa D_{\rho} / 4\right)^{1 / 2},
\end{aligned}
$$

where $D_{\rho}$ is the Drude weight of the optical conductivity (the charge stiffness), $v_{\rho}$ the velocity of the charge excitations, and $\kappa$ the compressibility. The use of three different relations for $K_{\rho}$ is important to verify the validity the calculation. In particular, the three relations will give inconsistent results if the system is not a LL, or if finite-size or other systematic errors are present.

The Drude weight can be calculated from QMC simulations as the $\beta \rightarrow \infty$ limit ob 36 . 37

$$
D_{\rho}=\pi\left[\langle-K / N\rangle-\Lambda_{c}(i 2 \pi / \beta)\right],
$$

where $K$ is the kinetic energy, and $\Lambda_{c}\left(i \omega_{m}\right)$ is the Matsubara frequency-dependent charge current-current correlation function:

$$
\Lambda_{\rho}\left(i \omega_{m}\right)=\frac{1}{N} \int_{0}^{\beta} d \tau e^{i \omega_{m} \tau}\langle j(\tau) j(0)\rangle .
$$

The standard method used in exact diagonalization calculations of the compressibility $\kappa$ employs a finite difference approximation involving ground state energies for different numbers of particles 16 explicitly,

$$
\kappa^{-1}=N_{e} \frac{\left[E\left(N, N_{e}+2\right)+E\left(N, N_{e}-2\right)-2 E\left(N, N_{e}\right)\right]}{2},
$$

where $E\left(N, N_{e}\right)$ is the ground state energy of $N_{e}$ electrons on an $N$-site lattice. The compressibility may be also computed from the $q \rightarrow 0$ limit of the static charge susceptibility;

$$
\kappa=\chi_{c}(q \rightarrow 0)
$$

where

$$
\chi_{\rho}(q)=\frac{1}{N} \sum_{j, l} e^{i q(j-l)} \int_{0}^{\beta} d \tau\left\langle n_{j}(\tau) n_{l}(0)\right\rangle,
$$

where $n_{j}(\tau)$ is the charge at site $j$ and imaginary time $\tau$. This definition avoids the errors due to discretization in the finite-difference definition (10), and we will therefore primarily use Eq. (11) here. Finally, the velocity $v_{\alpha}$ associated with the gapless charge $(\alpha=\rho)$ or spin $(\alpha=\sigma)$ mode may be extracted from the ratio 19

$$
W_{\alpha}(q)=2 S_{\alpha}(q) / \chi_{\alpha}(q),
$$

where $S_{\alpha}(q)$ is the static structure factor given by Eq. (3). $W_{\alpha}(q)$ gives an upper bound to the energy of the lowest excitation of momentum $q$ and becomes the exact excitation energy as $q \rightarrow 0$ in a LL, so that $v_{\alpha}$ can be directly extracted from the $q$-dependence for small $q$. Hence, the quantities needed for all three estimates of $K_{\rho}$ defined in Eqs. (7) can be computed directly from QMC data. Examining Eqs. (7b), (11), and (13), one can see that $K_{\alpha}$ is also given directly by the slope of the static structure factor as $q \rightarrow 0$ :

$$
K_{\rho, \sigma}=\frac{1}{\pi q} S_{\rho, \sigma}(q \rightarrow 0) .
$$

This relation may also be obtained directly from the calculation of the charge charge or spin-spin correlation functions in LL theory:15

$$
\left\langle n_{\alpha 0} n_{\alpha r}\right\rangle=-\frac{K_{\alpha}}{(\pi r)^{2}}+\frac{\cos \left(2 k_{F} r\right)}{r^{1+K_{\alpha}}}+\cdots
$$

The Fourier transform of the non-oscillating term of equation (15) leads to the expression (14) for $K_{\rho}$ and $K_{\sigma}$. As the structure factor is usually much easier to calculate numerically than the compressibility or the Drude weight, Eq. (14) is quite useful for calculating Luttinger liquid exponents. In particular, because $S_{\alpha}(q)$ only depends on equal-time correlations in imaginary time, for most QMC methods the structure factors converge much faster than $D_{\rho}$ or the susceptibilities and hence may provide a better estimate for $K_{\rho}$. But the important caveat applies that the consistency among all three relations must be checked, which requires more detailed calculations. Moreover, one additional consistency check is available in regions where one expects no spin gap. There it is required that $K_{\sigma}=1$, which can be verified (or disproved!) from the $q \rightarrow 0$ limit of $S_{\sigma}(q)$. Of course, the limit $q \rightarrow 0$ is impossible to attain strictly in numerical simulations of finite systems. Since Eq. (13) is an upper bound of the lowest excitation energy at momentum $q$, we expect that values of $K_{\rho}$ and $K_{\sigma}$ calculated from equation (14) to be in general slightly larger than their true values. Effects of non-linearity in the true lowest excitation energy should be smaller for typical values of the smallest $q$ accessible (i.e., we expect effect of the 
broadening of the mode to be larger than effects of nonlinearity).

One additional complication to using the slope of the structure factor to determine $K_{\rho}$ is that for very small fillings, $2 k_{F}$ may be close in momentum to the smallest $q=q_{1}$. A strong broad CDW peak at this momentum may then affect the behavior all the way down to $q_{1}$. The value obtained for $K_{\rho}$ will then still be an upper bound, but may be much larger than the true value.

Since from Eq. $(\sqrt{7 b})$ one sees that $K_{\rho}$ is proportional to the compressibility, $\kappa$, which (naively) should diverge at the phase separation boundary, calculations of $K_{\rho}$ using Eq. (7b) or equivalently Eq. (14) near a region of phase separation typically show a strongly increasing $K_{\rho}$. This increase of $K_{\rho}$ has often been interpreted (in a variety of one-dimensional models) as evidenceffr a r rgion of dominant superconducting correlations 8 , 8, 26, 17,22. A more careful analysis begins by noting that in an infinite system, $\kappa$ jumps discontinuously from a finite value to infinity at the phase separation boundary if the transition is first order, which is normally the case. However, as observed above ip Sec. II, and also recently by Hellberg and Manousakis, ${ }^{8} \kappa$ in a finite system can diverge only inside the region where the infinite system is phase separated, due to the energy cost of the interface between the two phases. Hence, a result $K_{\rho}>1$ based on a diverging $\kappa$ obtained from small systems may be misleading, since it is possible that the system is already phase separated in the thermodynamic limit.

As already noted, all these considerations raise legitimate concerns about the reliability of the previous calculations of the phase separation boundary in the EHM and hence also lead to concerns about whether $K_{\rho}$ exceeds mity in the uniform phase. Indeed, a recent QMC study 18.19 of a two-band 1D Hubbard model related to the EHM (a two-band "Cu-O" chain) showed that a firstorder phase separation transition preempts superconductivity in all but a small part of the phase diagram, in contrast to a previous ED study 17 which indicated that superconducting fluctuations always dominate in the neighborhood of phase separation.

\section{B. QMC results for $K_{\rho}$ in the EHM}

Using QMC techniques to evaluate $K_{\rho}$ accurately via all three Eqs. (7) is extremely time consuming, as $D_{\rho}$ in particular is difficult to calculate in the $V \gg U$ parameter region. Accordingly, in this section we first present results using all three relations (7) for only one value of $U$, and then use these detailed results to "benchmark" our alternate method of getting $K_{\rho}$ directly from Eq. 14. We choose $U=1$ for these calculations, and find excellent agreement between the two methods, which then justifies our use of the static structure factor method for other values of $U$.

In addition to the QMC simulations for 32-, 64-, and 128-site chains, we have performed Lanczös ED calculations for 16 sites, in order to investigate systematically the finite-size effects. We have also checked the QMC simulations against the ED data for this system size. In our ED calculations, we use Eq. (10) to define the compressibility $\kappa$ and to extract the charge velocity from the lowest charge excitation energy.

We begin with a comparison (for $U=1$ and varying $V$ ) of the various low-energy parameters used in calculating $K_{\rho}$. Figure 5 compares the Drude weight, the charge velocity, and the compressibility as computed for $n=1 / 2$ (quarter filling). Up to $V \approx 2$ the ED and $\mathrm{QMC}$ results agree almost perfectly. For larger $V$ the deviations are due to the larger finite-size effects in the ED data. For $V \lesssim 6$, the finite-size errors in the ED results seem to be greatest for $v_{\rho}$; hence we expect that Eq. (7d), which is the only Luttinger liquid relation not involving $v_{\rho}$, will give the best estimate for $K_{\rho}$ in small systems. For large $V$, the slower increase of the compressibility as computed by ED is likely primarily due to discretization errors in Eq. 10, which become large in regions where the energy curvature changes rapidly as a function of $N_{e}$ (as is the case close to phase separation).

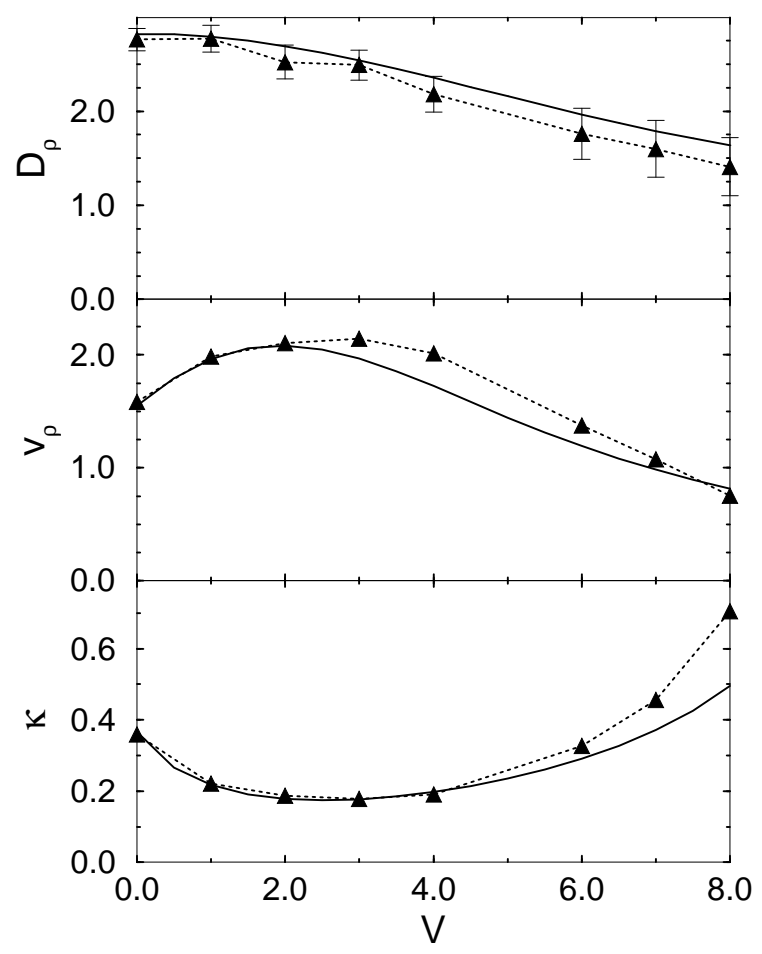

FIG. 5. Comparison of the Drude weight $\left(D_{\rho}\right)$, the charge velocity $\left(v_{\rho}\right)$, and the compressibility $(\kappa)$ for $U=1$ as computed by ED with $N=16$ (solid curves) and QMC with $N=64$ (triangles). Where not shown, the QMC error bars are approximately the size of the symbols (somewhat larger for $V=7,8)$. The dashed lines provided guides to the eye for the QMC data.

Figure 7 shows results for $K_{\rho}$ as a function of $V$ calculated for $U=1$ by Lanczös diagonalization and QMC 
simulations. The data points based on Eq. (7b) are equivalent to using only the structure factor in determining $K_{\rho}$ according to Eq. (14). The error bars of the other methods are not shown for clarity; in general they are at least twice as large as the errors using the structure factor slope method. Further, in Figure 7 , the $K_{\rho}$ values for $V=8$ calculated using Eqs. (7a) and (7c) have been left off, as their statistical errors are too large for this large $V$. Given the equivalence of using Eq. (7b ) to the calculation of the slope of the structure factor at $q=0$, it is clear that the slope method provides a good estimate for $K_{\rho}$, as it agrees with the other methods within error bars but shows aller statistical fluctuations. Previous $K_{\rho}$ calculations 10 using ED methods had seen large differences among the different relations (77). As seen in Fig. 7, these differences are greatly reduced using larger lattices. Indeed, our QMC results on 64-site systems show that the three relations give equivalent results, to within error bars. Most importantly, all the $K_{\rho}$ values obtained for $V<8$ and $U=1$ are less than one. The Maxwell construction, shown in Fig. 3, indicates that phase separation occurs at quarter filling for $V$ only slightly larger than $V=8$. It is therefore clear that there is no extended region where $K_{\rho}>1$ before phase separation, although there is a definite increase as the phase separation boundary is approached. We can of course not strictly rule out the existence of an extremely narrow region where $K_{\rho}$ exceeds one. However, this seems unlikely in view of our results for the spin susceptibility at $V=8$ and 10 shown in Figure 6. In both cases there is a clear peak at $q=2 k_{\mathrm{F}}$, which would not be expected if $K_{\rho}$ exceeds unity. Since our Maxwell construction indicates that the $V=10$

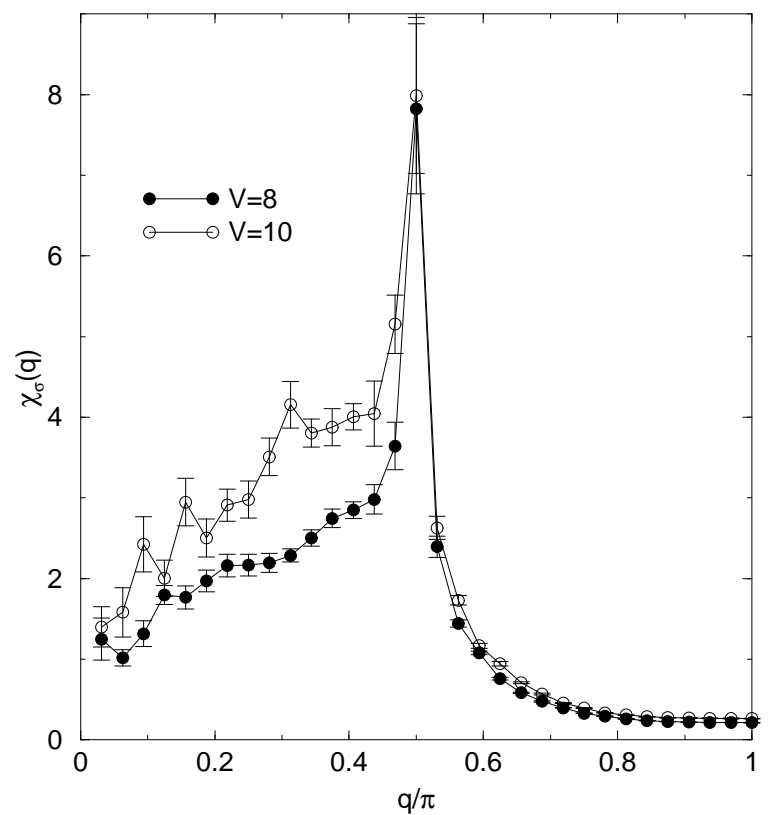

FIG. 6. $\chi_{\sigma}$ for $U=1$ and $n=0.5$ (quarter-filling) at $V=8$ (solid circles) and $V=10$ (open circles).

system should be phase separated in the thermodynamic limit, we can conclude that the dominant SDW fluctuations persist at the phase separation boundary and there is no region with $K_{\rho}>1$.

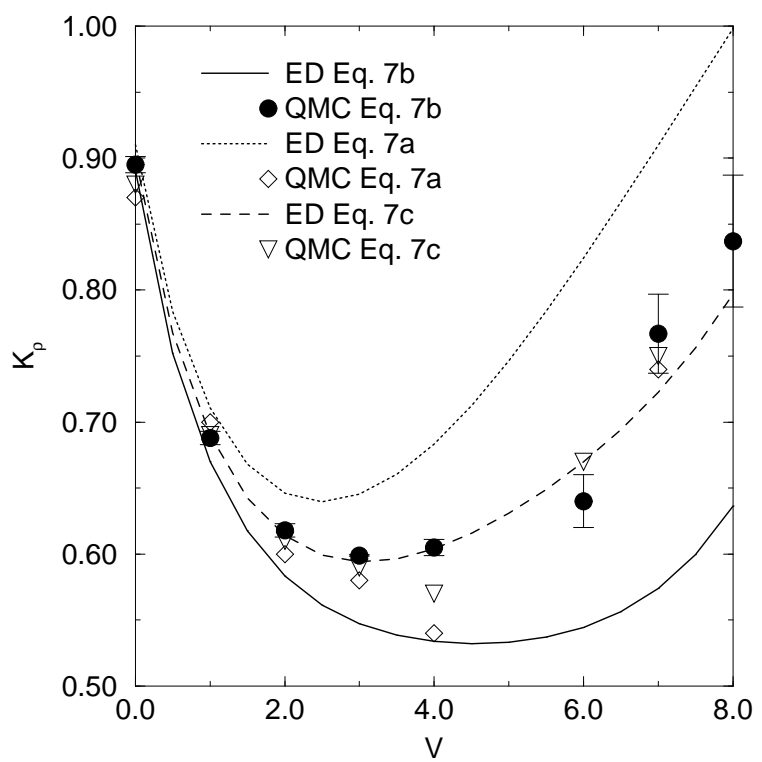

FIG. 7. $K_{\rho}$ vs. $V$ for $U=1$ calculated using all Eqs. (7). ED results (curves) for 16 sites are compared with QMC results for 64 sites (symbols). Error bars are shown only for Eq. (7b) for clarity; error bars for Eqs. (7a) and (7d) are larger. Phase separation for this filling and $U$ occurs at $V \approx 8$

For other fillings and $U$ we have concentrated on $V=8$. For $1<U<4$ we find similar behavior in $K_{\rho}$ (based on the slope of $S_{\rho}(q)$ for 32 and 64 site systems) to the $U=1$ case: $K_{\rho}$ increases as the density increases, but does not exceed one before phase separation. For $-3<U<0$, our determination of the phase separation boundary is not as accurate, and in some cases calculations for for 32- and 64-site systems give $K_{\rho} \gtrsim 1$ in regions where there are no clear signals of phase separation. However, in cases where we have also carried out calculations for $N=128$, the general trend seems to favor phase separation over a stable uniform state with $K_{\rho}>1$ (recall the data for $U=-1$ and $U=0$ at $n=9 / 16$ in Fig. (1).

The persistence of finite-size effects in calculations of $V_{\mathrm{PS}}$ and $K_{\rho}$ close to phase separation for systems as large as $N=64$ sites emphasizes the importance of studying and understanding finite-size effects in calculations of $K_{\rho}$ and related quantities. In Sec. V we will discuss the complete phase diagram of the model and comment further on the behavior of $K_{\rho}$ as $V \rightarrow V_{\mathrm{PS}}$.

\section{Unusual charge correlations in the EHM}

Luttinger liquid theory predicts structure in the charge or spin response only for multiples of $2 k_{F}$, which is a consequence of the low-energy effective model being lin- 
earized about $\pm k_{F}$. Peaks are typically seen only at $q=2 k_{\mathrm{F}}$ and/or $q=4 k_{\mathrm{F}}$ (only for the charge if there is a spin gap). The $2 k_{\mathrm{F}}$ peaks should diverge as $T \rightarrow \Omega$ if $K_{\rho}<1$, and $4 k_{\mathrm{F}}$ peaks are divergent for small $K_{\rho} .15$ Divergences in finite systems can of course be seen only as $N \rightarrow \infty$.

In studying the $V \gg U$ region of the EHM, we have found strong charge response peaks also at wavevectors that are not multiples of $2 k_{F}$; we shall use the term "anomalous peaks" to refer to these unusual charge correlations. Importantly, however, these anomalous peaks appear to be associated with gapped modes. As the temperature decreases and $N$ increases they do not diverge. Hence we believe the system is still a LL in this region for sufficiently low energies. We shall comment further below on the interpretation of these anomalous peaks.

Figure 8 shows the charge susceptibility for several fillings for $V=8$ and $U=-1,0,1$. Starting with the $U=1$ data, we see that at small fillings the susceptibility is dominated by a large peak at $q=4 k_{F}$. This corresponds to a system with almost no pairs, where the particles behave essentially as spinless fermions. For higher fillings the large peak shifts from the value of $4 k_{\mathrm{F}}$ to a slightly lower momentum - which for later purposes we call $4 k_{\mathrm{F}}^{\mathrm{eff}}$ - whose value depends on the filling as well as the other model parameters. As one moves further into the phaseseparated

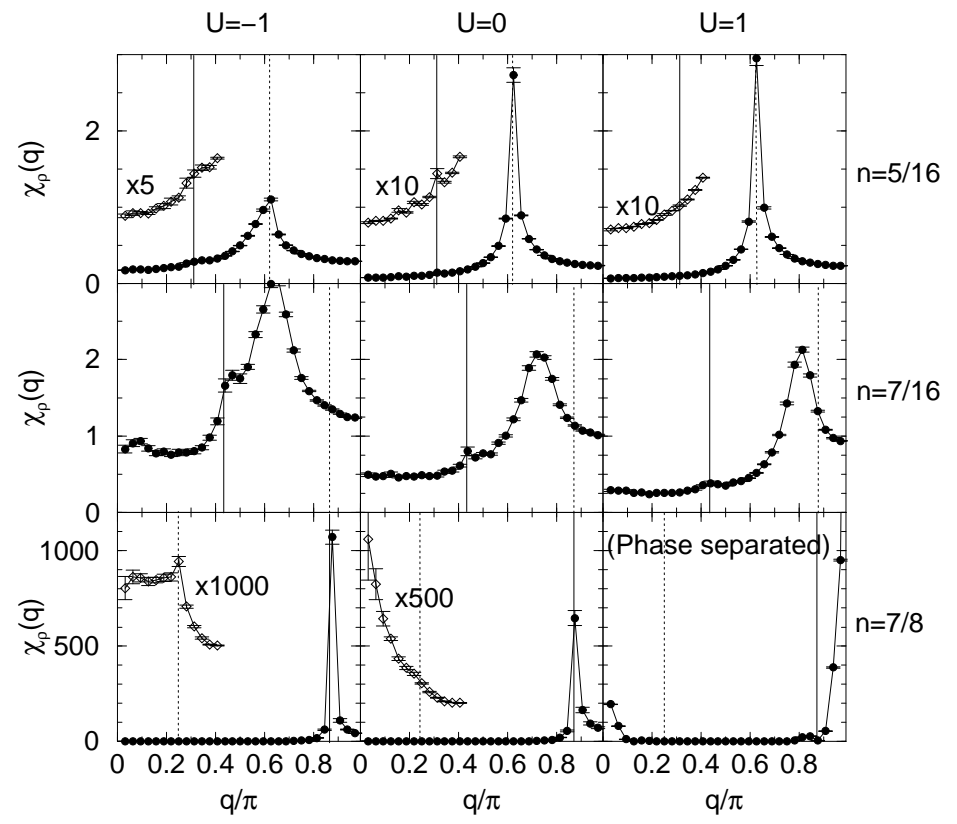

FIG. 8. Charge susceptibility for $V=8, U=-1,0,1$. All data are for 64 site systems. The vertical solid and dashed lines indicate $q=2 k_{\mathrm{F}}$ and $q=4 k_{\mathrm{F}}$, respectively. Note that for $n>1 / 2,4 k_{F}=\pi(2-2 n)$ in the reduced zone scheme. Lines between data points are guides to the eye.

region (above $n \approx 5 / 8$ ) a strong peak develops at $q=\pi$, independent of $n$ (and hence $k_{F}$ ). This corresponds to the wave-vector of the high-density phase in the phase- separated region, which is a CDW state in which double occupancies alternate with empty sites.

Turning to the cases for $U=-1$ and $U=0$, the behavior is quite similar for low and intermediate densities. As in the $U=1$ case, at low fillings one sees a large $4 k_{\mathrm{F}}$ peak and a much weaker (but still observable in the $U=0$ data) $2 k_{F}$ peak. For intermediate fillings near the phase separation boundary, the main peak is no longer at $4 k_{\mathrm{F}}$ but rather at $4 k_{\mathrm{F}}^{\mathrm{eff}}$ as for $U=1$. This is shown clearly in the $U=0$ data for $n=7 / 16$, which is outside of the phase-separated region $\left(n<n_{\mathrm{LD}}\right)$. At large fillings $\left(n>n_{\mathrm{HD}}\right)$, instead of the peak at $q=\pi$ seen in the $U=1$ data, the $U=-1$ data shows a strong peak at $2 k_{\mathrm{F}}$, reflecting the re-entrance (as a function of $n$ ) of the homogeneous phase, which is characterized by $2 k_{\mathrm{F}} \mathrm{CDW}$ fluctuations. A weaker $4 k_{\mathrm{F}}$ peak can also be seen at this filling. For $U=0, n=7 / 8$ is very close to our estimated re-entrance point $\left(n_{\mathrm{HD}} \approx 0.88\right)$.

Figure 9 shows the location of the dominant peak in the charge susceptibility as a function of filling for $V=8$ and $U=-1,0,1$. For small fillings, all the systems are dominated by the $4 k_{F}$ charge response. As the filling is increased, the anomalous $4 k_{\mathrm{F}}^{\mathrm{eff}}$ peak dominates the response for these system sizes. For still higher fillings the normal $2 k_{\mathrm{F}}$ peak dominates. As already noted, we see a $2 k_{\mathrm{F}}$ peak also at the densities where the anomalous peak is present (see Figs. 8 and 9), and we expect it to

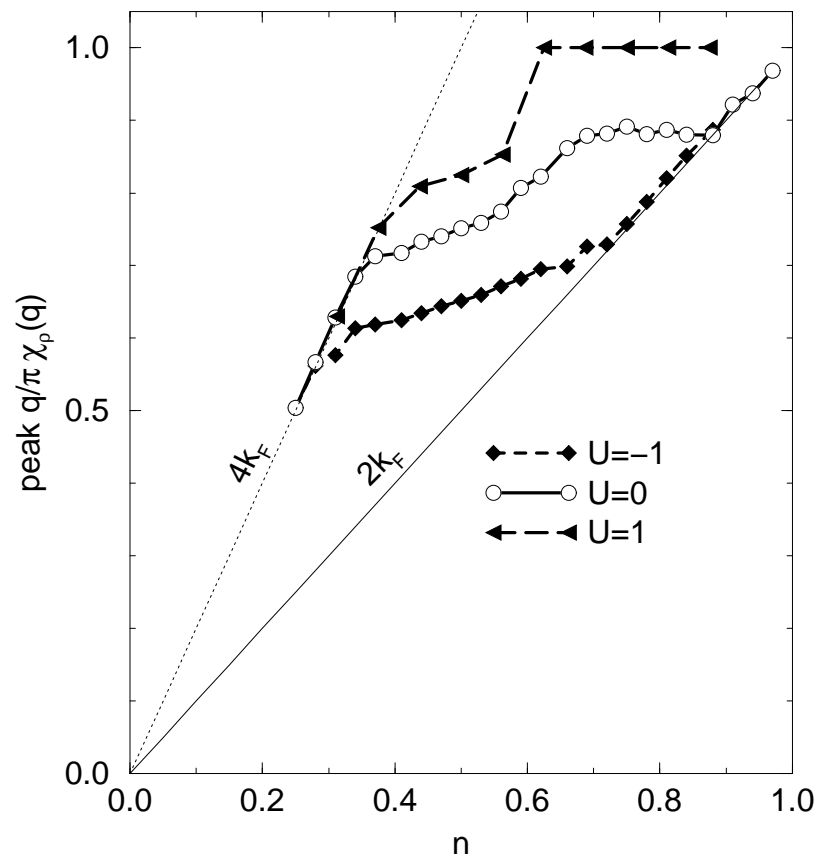

FIG. 9. Momentum value of the largest peak in the charge susceptibility $\chi_{\rho}(q)$ for a 64 -site system as a function of band filling $n$ for $V=8, U=1,0,-1$. The peak position was determined by fitting a $2^{\text {nd }}$ degree polynomial to points near the peak. The solid and dotted lines indicate $q=2 k_{F}$ and $q=4 k_{F}$ respectively.

diverge as $N \rightarrow \infty$ and $T \rightarrow 0$, reflecting asymptotic LL 
behavior. Note the constant behavior of the peak position for $U=0$ at $0.65 \lesssim n \lesssim 0.88$; this indicates that this peak originates from the high-density phase of the phaseseparated system, which apart from growing in size as $n$ increases remains unchanged in the phase separation region. This behavior is seen less clearly for $U=-1$, where the region of phase separation is apparently very narrow. Close to half filling a uniform state again stabilizes for $U=-1,0$, whereas the $U=1$ system remains phase separated all the way up to half filling.

Since the anomalous charge response is not at a wavevector possible within the Luttinger liquid formalism, it is important to determine whether the corresponding $4 k_{\mathrm{F}}^{\text {eff }}$ peak diverges with decreasing temperature and thus has thermodynamic relevance in the low-energy sector. Figure 10 shows the momentum dependence of the charge susceptibility at three different temperatures for a parameter set where the anomalous charge fluctuations are strong. The inset shows the peak value versus inverse temperature for two different system sizes. We see that as the temperature is lowered, the anomalous peak does not diverge (it in fact has a maximum at a finite temperature), and there is almost no dependence on $N$.

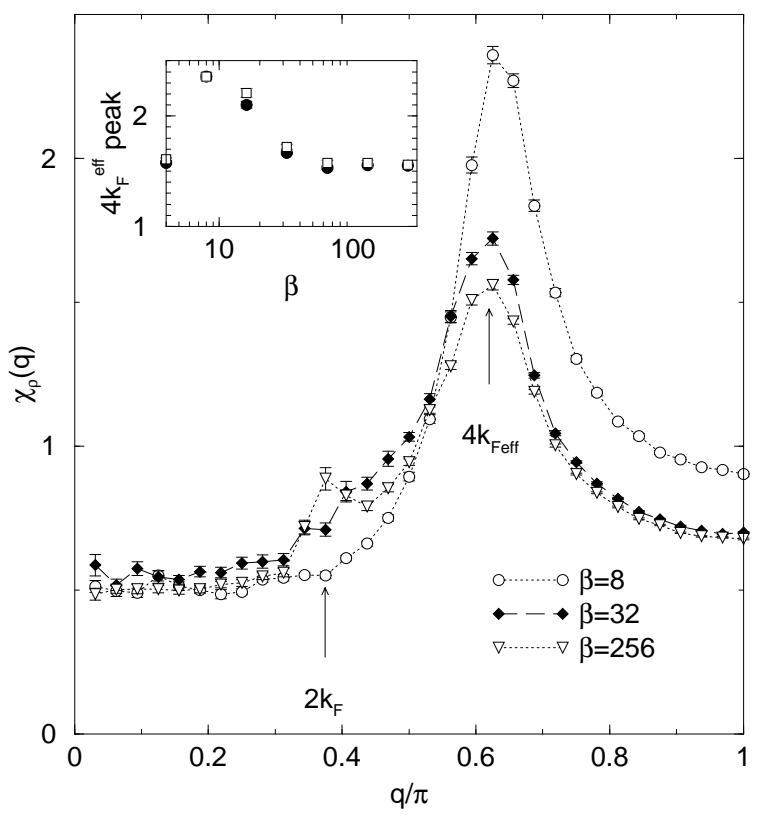

FIG. 10. Behavior of non-LL "anomalous" $4 k_{\mathrm{F}}^{\mathrm{eff}}$ charge peak in $\chi_{\rho}(q)$ for $V=8, U=-1, n=3 / 8$ vs. temperature. The symbols connected by dashed lines in the main figure are for 64 -site systems. The inset shows the peak value vs. $\beta$ for 32 -site (circles) and 64-site (squares) systems. Note also the presence of a normal $2 k_{\mathrm{F}}$ peaks which grows with increasing $\beta$

Nonetheless, this non-LL charge peak is still relatively low in energy. Using equation (13) we are able to estimate an upper bound of $E \sim 0.75 t$ above the ground state at $V=8, U=-1$ and $n=3 / 8$. In Figure 10, one clearly sees that the normal $2 k_{F}$ peak indeed grows with decreasing temperature, as expected in a Luttinger liquid with $K_{\rho}<1$, although the amplitude is relatively weak even at $\beta=256$.

We believe that the anomalous charge response is due to on-site pairs that are sufficiently long-lived (due to the difficulty of breaking them up via high-energy intermediate states) to form a CDW together with the single particles of the system. Specifically, in the infinite $V$ effective model of Penc and Mila, there are two kinds of particles; spinless fermions and bosons with charge two. The number of doubly occupied sites in the simulation of the EHM corresponds to the number of bosons, and hence we can calculate the average total number of particles (fermions plus bosons) within the effective model. We find that the momentum of the anomalous charge peak is consistent with spacing uniformly a number of particles equal to this total the number of particles. Hence, there is a tendency to form a CDW consisting of a mixture of fermions and bosons which repel each other. Nevertheless, the non-divergence of the anomalous peak, the presence of a $2 k_{F}$ peak, and the consistency among the Luttinger liquid relations indicate that the asymptotic low-energy properties are still those of spin- $1 / 2$ fermions forming a LL.

\section{Spin gap in the EHM}

Our results for the spin susceptibility of the extended Hubbard model in the $V \gg|U|$ region show either a

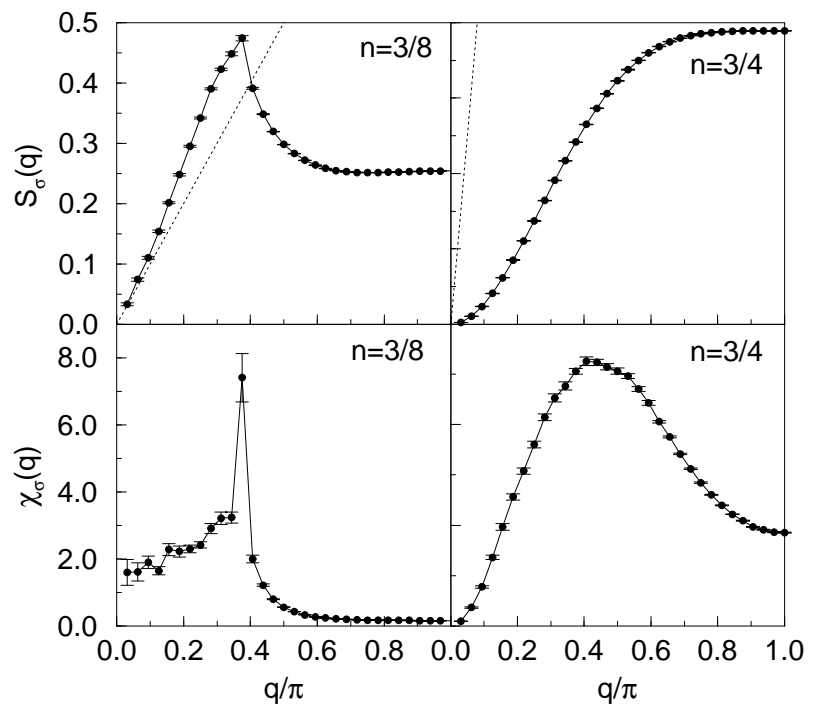

FIG. 11. $\chi_{\sigma}(q)$ and $S_{\sigma}(q)$ comparing spin-gapped to non-gapped cases. Left panels are for $V=8, U=-1$, and $n=0.375$, where no gap is present. Right panels are for same $U$ and $V$ but $n=0.75$, where the system is gapped. Solid curves connecting data points are guides to the eye. The dotted lines indicate slope $\pi$ for $S_{\sigma}(q)$ vs. $q$.

normal Luttinger liquid $2 k_{F}$ peak or the presence of a spin gap. In general, we find that the spin response is 
much noisier and harder to converge numerically than the charge response, as expected in a parameter region dominated by charge correlations. To determine whether the system is spin gapped, we examine the spin susceptibility $\chi_{\sigma}(q)$ in the limit $q \rightarrow 0$. If $\chi_{\sigma}(q \rightarrow 0)=0$, the system is spin gapped. The presence of a spin gap can also be inferred from the structure factor $S_{\sigma}(q)$ : If there is no spin gap, then $K_{\sigma}=1$, which translated into a slope $\pi$ for the structure factor versus $q$ (as discussed in Sec. IVA). In a spin-gapped system $S_{\sigma}(q)$ should approach zero faster than linearly and in particular should fall below the line with slope $\pi$ (instead of approaching this line from above, as expected if there is no spin gap). Again, one must use care in phase-separated regions, as the $\chi_{\sigma}(q)$ will show a mixture of responses from both phases. Figure 11 shows examples of $\chi_{\sigma}(q)$ and $S_{\sigma}(q)$ for parameters with and without a spin gap. The two different behaviors discussed above can be clearly discerned.

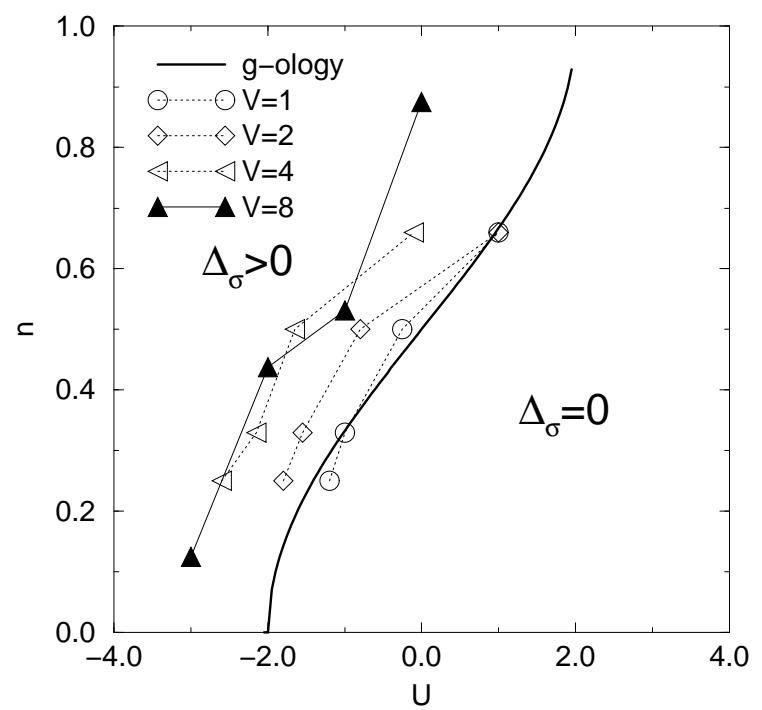

FIG. 12. Summary of spin gap boundary. The solid curve is the g-ology result for $V=1$. Hollow symbols connected by dotted lines are results of ED calculations and finite-size scaling for the values of $V$ indicated. Solid symbols connected by lines are QMC results for 32 or 64 sites.

When interpreting the complete ground state phase diagram, it is useful to consider the development of the spin gap boundary as $V$ increases from $V=0$. For the case $V=0$, there is a spin gap (and dominate singlet superconducting fluctuations) for all negative $U$. For weak coupling, g-ology results may be used, and they predict a spin gap for $g_{1}=U+2 V \cos (\pi n)<0$. For larger $U$ and $V$ we have used both QMC and Lanczös ED. In the ED calculations, the gap was computed from the ground state energies of the system, and the system with one spin flipped: $\Delta_{s}=E\left(N, n_{\uparrow}, n_{\downarrow}\right)-E\left(N, n_{\uparrow}+1, n_{\downarrow}-1\right)$. For each filling, two or three system sizes and finite-size scaling of the gap values versus $1 / N$ were used. The $\mathrm{QMC}$ estimate for the spin gap boundary was extracted from structure factor results such as those shown in Fig. 11.
We consider a system to be gapped in cases where the $S_{\sigma}(q)$ curve falls below the line with slope $\pi$ as $q \rightarrow 0$. The QMC and ED results are combined in Figure 12 . One can see that (i) for weak interactions the ED results are quite close to the g-ology predictions; and (ii) importantly, as $V$ increases, the spin-gap boundary line moves towards the infinite $V$ result of $U=-4$.

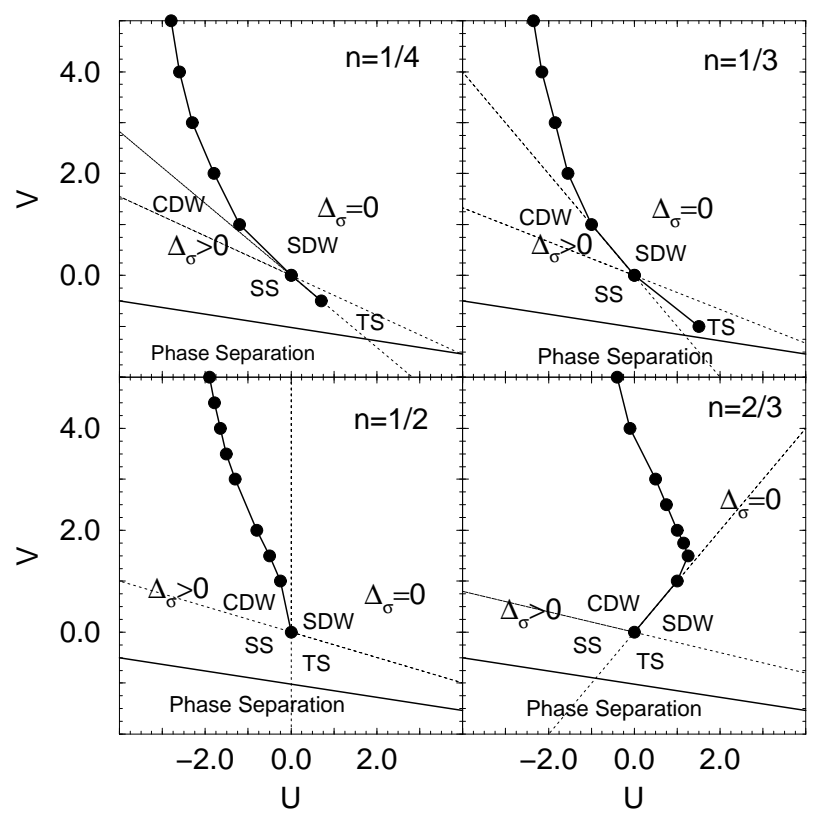

FIG. 13. The spin gap region as a function of $U$ and $V$ for $n=1 / 4, n=1 / 3, n=1 / 2$, and $n=2 / 3$. The dotted lines indicate the phase boundaries from g-ology, and the solid circles are the results of our exact diagonalizations. The spin gap is present to the left of the boundaries shown. The approximative 㓩se separation boundaries for negative $U$ calculated in Refs 10 are also shown.

For purposes of comparison to previous results on the spin gap in the parameter region $V>0$, 8 we present ED results graphed in the $(U, V)$ plane for various fillings in Figure 13. In this figure we also show the gology predictions for the dominant fluctuations, as well as the phase separation boundafy for the $V<0$ regime, which was calculated previously. 0 Our spin gap boundary for $n=1 / 2$ agrees closely with previous numerical work presented by PM. However, our boundary for $n=2 / 3$ is significantly different from that obtained previously, 8 with our results placing the spin gap boundary further towards the negative- $\mathrm{U}$ side of the phase diagram.

\section{DISCUSSION AND CONCLUSION}

In the preceding sections, we have explored numerically the $V \gg|U| \sim 1$ parameter region of the $1 \mathrm{D}$ extended Hubbard model for a wide range of band fillings. Our numerical approaches included three different forms of QMC simulations on systems of up to 128 sites and 
Lanczös exact diagonalizations and finite-size scaling for systems of up to 16 sites. In addition, we relied on some analytic results for $V \rightarrow \infty$.

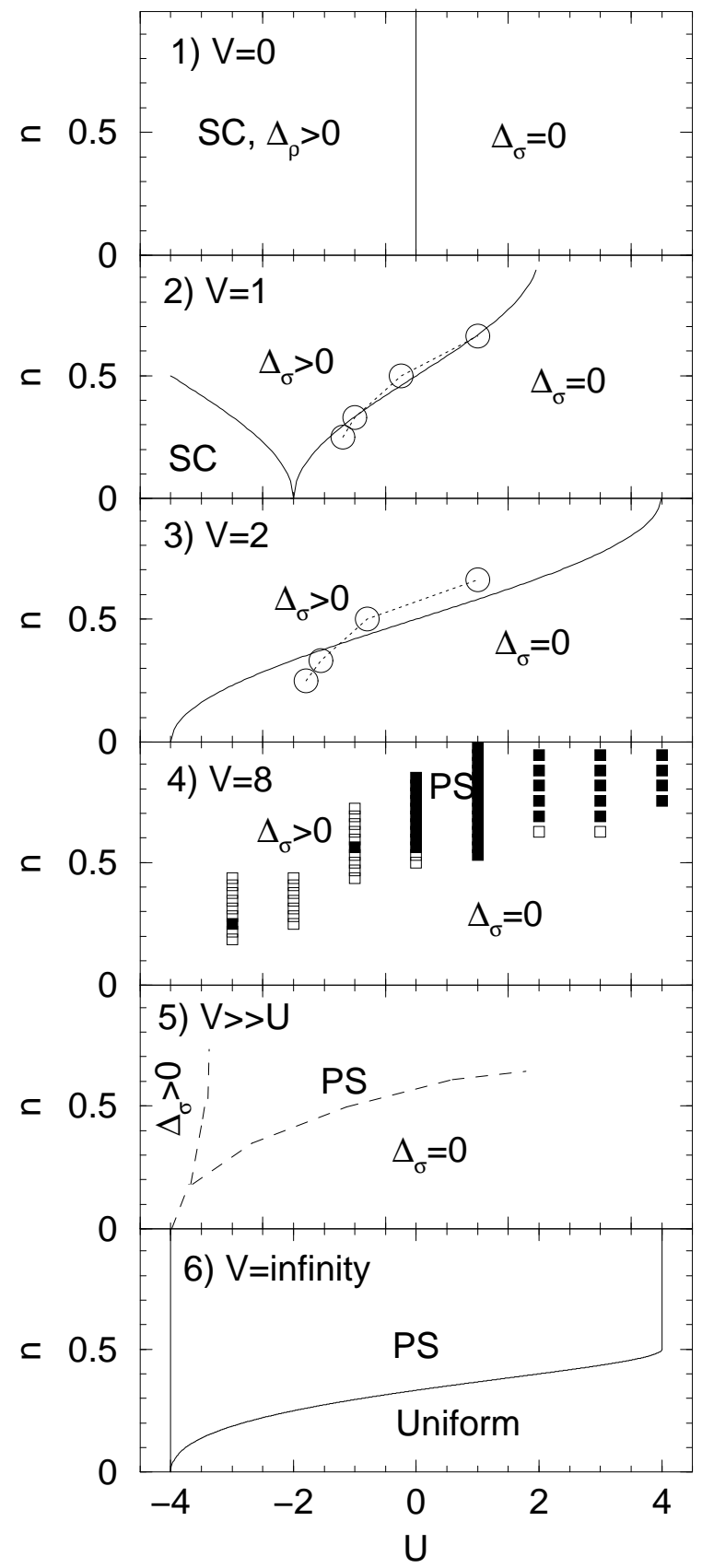

FIG. 14. Phase diagram of EHM in the region $-4<U<4$. Circles denote exact diagonalization data. The curves for $V=0, V=1$, and $V=2$ are from g-ology. At $V=8$, filled boxes are phase separation points we have confirmed [via a Maxwell construction or the $q \rightarrow 0$ behavior of $S_{\rho}(q)$ using $\mathrm{QMC}]$. Open boxes are also most likely correspond to phase separation, but our results here are less certain. The full phase-separated region is inside a curve enclosing all these points. The curves shown in panel 5 are only schematic. The curves in panel 6 are the exact solution for $V \rightarrow \infty$
A complete quantitative mapping of the phase diagram even for the restricted region $V \gg|U| \sim 1$ would require enormous numerical work to determine precisely all phase boundaries for many values of the parameters. Although we have not carried out such a program in the present study, we believe that we have developed an accurate qualitative (with quantitative results for specific parameters) picture of the phase diagram in a parameter region of the EHM about which previously little has been understood. The considerable complexity we have found in the model serves to illustrate the great care that must be taken in interpreting numerical data, in particular those used as signals of superconductivity and phase separation in exact diagonalization results for small lattices.

Our most important conclusion is that phase separation extends to much lower values of $V$ than previously reported. As a result, the Luttinger liquid exponent $K_{\rho}$ does not exceed one before phase separation, and hence the ground state is not dominated by superconducting fluctuations. This resolves the difficulties in interpretations of previous exact diagonalization results, 6 which indicated an extended region of gapless spin excitations and $K_{\rho}>1$, which taken together would correspond to dominant triplet pairing correlations. Since the naive picture of superconductivity in the $V \gg|U|$ region involves singlet on-site pairs, one would rather have expected a spin gap to accompany $K_{\rho}>1$. Our results explain this puzzling result as simply due to difficulties in detecting phase separation in small systems. It should be noted that our results for $K_{\rho}$ in the uniform phase agree quite well with the previous estimates 6 - up to our calculated phase separation boundary.

To summarize our results for the EHM phase diagram for the $V \gg|U|$ region, it is most useful to examine how the features (phase separation, spin gap, possible superconductivity, etc., evolve together from the more familiar $V \sim 0$ region toward $V=\infty$. Further, since exact results exist in both the $V=0$ and $V=\infty$ limits, following the evolution of the various boundaries among the phases from the two known solutions is very helpful in understanding the global evolution of the phase diagram. Figure 14 combines our numerical data, the exact limiting cases, and qualitative considerations to summarize our results. The numbered comments below correspond to the panels of figure 14, labeled from top (1) to bottom (6):

1. $(V=0)$ The EHM here reduces to the standard Hubbard model and is spin gapped with dominant superconducting fluctuations for all $U<0$.

2. $(V=1)$ As $V$ increases from zero, the single (vertical) boundary that for $\mathrm{V}=0$ separates the superconducting/spin-gapped phase from the Luttinger liquid/non-spin-gapped splits into two separate boundaries, producing a central spin-gapped but non-superconducting region, which extends into both positive and negative $U$. A region of 
singlet superconductivity is also present. Results of g-ology agree well with exact diagonalization at this weak coupling.

3. $(V=2)$ For this intermediate value of $V$, the "standard" superconducting region has disappeared from our parameter region, moving to larger negative $U$ values. The spin gap region also recedes further towards negative $U$.

4. $(V=8)$ At large $V$, a region of phase separation has entered. Based on the movement of the phaseseparated region towards negative $U$ as $V$ increases, phase separation probably first enters near the end of the spin gap line, i.e. near $n=1$ and between $U=0$ and $U=4$. For $U<0$, the spin gap boundary remains largely unchanged from weak coupling. At positive $U$, the high-density phase consists of pairs on every other site. For negative $U$, the highdensity phase expands slightly, as the dominant wavevector is no longer at $q=\pi$. In addition, the region of phase separation exists over a limited region of $n$ and vanishes near half filling, with the homogeneous phase re-entering. For small fillings, $4 k_{F}$ charge correlations dominate, while near half filling $2 k_{F}$ charge correlations dominate. At intermediate fillings we find an anomalous, nonLuttinger liquid peak that is non-divergent with increasing system size or decreasing temperature. This peak appears to reflect CDW fluctuations involving long-lived pairs that repel each other as well as the unpaired particles. Our data, which clearly show phase separation at this $V$, show no convincing signs of dominant superconducting correlations in the uniform phase, leading us to conclude that any region of superconductivity for $V \gg|U|$ is very small indeed, or, more likely, does not exist.

5. $(V \gg U)$ At still larger $V$, we expect the region of phase separation to grow towards the point $U=$ $-4, n=0$. We show a schematic phase diagram in this case.

6. $(V=\infty)$ The point connecting the spin gap and phase separation boundaries has moved to $(U, n)=$ $(-4,0)$. The spin gap boundary is now the vertical line from $n=0$ to $n=1$ at $U=-4$. The phase separation region consists of either effective spinless fermions or pairs, and the high density phase has pairs on every other site.

One may argue that our numerical data cannot exclude the existence of an extremely narrow strip of superconductivity preceding phase separation. However, in the non-spin-gapped region this seems unlikely in view of the obvious difficulty in accounting for dominant triplet superconducting fluctuations in the $V \gg|U|$ region. The exact $V \rightarrow \infty$ solution also provides a counter-argument: below the phase separation boundary $\left(n<n_{\mathrm{LD}}\right)$, the system is mapped onto spinless fermions, and the dominant correlations are then clearly not superconducting in the neighborhood of the phase separation boundary. For large but finite $V$, we find numerically that $K_{\rho}$ does not appear to exceed unity close to the phase separation boundary. We instead find strong $2 k_{\mathrm{F}}$ spin fluctuations that do not vanish as the phase separation boundary is crossed. Hence we believe that the system is dominated by SDW fluctuations adjacent to the phase separation boundary in cases where there is no spin gap. On the other hand, for $U<0$ we find $K_{\rho} \approx 1$ in a narrow region for which we cannot definitely conclude that the system is phase separated. This ambiguous region coincides with our calculated spin gap boundary, and is essentially the region of the open boxes in panel 4 of Fig. 14. Hence, if superconducting fluctuations indeed dominate in this region, they would be of the singlet type, which is what one would expect. However, also in this case it appears more likely that $K_{\rho}$ does not exceed unity at the phase separation boundary. The $V \rightarrow \infty$ case again provides support for this result: On the left-hand side of the phase-separated region shown in panel 6 of Fig. 14 (i.e., $U<-4$ ), the system contains only pairs, and for large but finite $V$ exhibits strong $2 k_{\mathrm{F}}$ CDW fluctuations. Dominant superconducting fluctuations do appear in this region for small values of $V$ but are replaced by CDW fluctuations for moderate $V$ (see panels 2 and 3 of Fig. 14). It then appears unlikely that a small region of dominant superconducting fluctuations in the region $U \sim(-3,-1)$ and $n \sim(0.2,0.5)$ would re-appear as $V$ is increased and then again vanish as $V \rightarrow \infty$, but we do not have data that can definitely exclude this scenario.

Although we believe our results establish that phase separation, rather than superconductivity, dominates the $V \gg|U|$ region of the EHM, there remain a number of interesting open questions about some of the "exotic" non-Luttinger liquid effects we have observed. In particular, two such questions involve (i) the exact nature of the state corresponding to the anomalous $4 k_{F}^{e f f}$ peak in the charge susceptibility and (ii) a more detailed understanding of the "re-entrant" phase separation behavior compared to the normal $U>0$ phase separation, where the high-density phase is at (the naively expected) halffilled density. We have presented qualitative interpretations of these "exotic" effects, but they may warrant further study, focusing on when they can appear, what simple effective model (if any) can be used to describe them, the relation between phase separation and the nonLuttinger liquid peak, and how this peak evolves with system size and temperature. One possible method to access this parameter region would be to study numerically an effective model including paired and single electrons similar to PM's $V \rightarrow \infty$ effective model. Such an effective model would eliminate electrons on nearest-neighbor sites, which could be additionally taken into account perturbatively, if necessary. We are currently exploring this approach. 
We are grateful to Steve Hellberg, Steve Kivelson, and Reinhard Noack for useful discussions. This work is supported by the NSF under grant DMR-97-12765. RTC acknowledges support of a NSF GRT fellowship. The numerical calculations were performed in part at the NCSA.

${ }^{1}$ H. G. Keiss (ed.), Conjugated Conducting Polymers, (Springer-Verlag, Berlin, 1992).

${ }^{2}$ T. Ishiguro and K. Yamaji, Organic Superconductors, (Springer-Verlag, Berlin, 1990).

${ }^{3}$ E. H. Lieb and F. Y. Wu, Phys. Rev. Lett. 20, 1445 (1968).

${ }^{4}$ D. Guo, S. Mazumdar, S. N. Dixit, F. Kajzar, F. Jarka, Y. Kawabe, and N. Peyghambarian, Phys. Rev. B 48, 1433 (1993).

5 J. E. Hirsch and D. J. Scalapino, Phys. Rev. B 27, 7169 (1983); 29, 5554 (1984).

${ }^{6}$ F. Mila and X. Zotos, Europhys. Lett. 24, 133 (1993).

${ }^{7}$ K. Penc and F. Mila, Phys. Rev. B 49, 9670 (1994).

${ }^{8}$ K. Sano and Y. Ono, J. Phys. Soc. Jpn. 63, 1250 (1994).

${ }^{9}$ H. Q. Lin, E. R. Gagliano, D. K. Campbell, E. H. Fradkin, and J. E. Gubernatis, in Proceedings of the 1993 NATO ARW on "The Physics and Mathematical Physics of the Hubbard Model", edited by D. Baeriswyl et al., (Plenum, New York, 1995)

${ }^{10}$ H. Q. Lin, E. R. Gagliano, D. K. Campbell, E. H. Fradkin, and J. E. Gubernatis, submitted to Phys. Rev. B.

${ }^{11}$ F. D. M. Haldane, Phys. Rev. Lett. 45, 1358 (1980).

12 J. M. Luttinger, J. Math. Phys., 4, 1154 (1963).

13 S. Tomonaga, Prog. Theor. Phys., 5, 544 (1950).

${ }^{14}$ A. Luther and V. J. Emery, Phys. Rev. Lett. 33, 589 (1974).

15 H. J. Schulz, Phys. Rev. Lett. 64, 2831 (1990).

${ }^{16}$ M. Ogata and M. U. Luchini and S. Sorella and F. F. Assaad, Phys. Rev. Lett. 66, 2388 (1991).

17 W. Barford and E. R. Gagliano, Physica B 194, 1455 (1994).

18 A. W. Sandvik and A. Sudbø, Phys. Rev. B 54, R3746 (1996).

19 A. W. Sandvik and A. Sudbø, Europhys. Lett. 36, 443 (1996).

20 A. Sudbø, C. M. Varma, T. Giamarchi, E. B. Stechel, and R. T. Scalettar, Phys. Rev. Lett. 70, 978 (1993); E. B. Stechel, A. Sudbø, T. Giamarchi, and C. M. Varma, Phys. Rev B 51, 553 (1995).

${ }^{21}$ E. Dagotto, J. Riera, Y. C. Chen, A. Moreo, A. Nazarenko, F. Alcaraz, and F. Ortolani, Phys. Rev. B 49, 3584 (1994).

${ }^{22}$ V. J. Emery and S. A. Kivelson and H. Q. Lin, Phys. Rev. Lett. 64, 475 (1990); Phys. Rev. 426523 (1990).

${ }^{23}$ A. W. Sandvik and J. Kurkijärvi, Phys. Rev. B 43, 5950 (1991).

24 A. W. Sandvik, J. Phys. A 25, 3667 (1992).

${ }^{25}$ D. C. Handscomb, Proc. Cambridge Philos. Soc. 58, 594 (1962); 60, 115 (1964).

${ }^{26}$ N. V. Prokof'ev, B. V. Svistunov, and I. S. Tupitsyn, preprint (cond-mat/9703200); Zh. Eks. Teor. Fiz. 64, 853
(1996) (translation in cond-mat/9612091).

27 A. W. Sandvik, R. R. P. Singh, and D. K. Campbell, Phys. Rev. B 56, 14510 (1997).

28 A. W. Sandvik, Phys. Rev. B 56, 11678 (1997); preprint (1998).

${ }^{29}$ H. G. Evertz, G. Lana, and M. Marcu, Phys. Rev. Lett. 70, 875 (1993); B. B. Beard and U. -J. Wiese, Phys. Rev. Lett. 77, 5130 (1996); J. -K.Kim and M. Troyer, Phys. Rev. Lett. 80, 2705 (1998).

${ }^{30}$ S. Zhang and J. Carlson and J. E. Gubernatis, Phys. Rev. Lett. 74, 3652 (1995).

${ }^{31}$ S. Zhang and J. Carlson and J. E. Gubernatis, Phys. Rev. 55, 7464 (1997).

32 J. Bonca, J. E. Gubernatis, M. Guerrero, E. Jeckelmann, and S. R. White, preprint, cond-mat/9712018.

${ }^{33}$ For a recent review, see, e.g., J. Voit, Rep. Prog. Phys. 57, 977 (1994).

34 J. Sólyom, Advances in Physics, 28, 201 (1979).

${ }^{35}$ V. J. Emery "Theory of the One-Dimensional Electron Gas" in Highly Conducting One-Dimensional Solids, ed. J. T. Devreese, pp 247-303, Plenum, New York 1979.

${ }^{36}$ W. Kohn, Phys. Rev., 133, A171 (1964).

37 D. J. Scalapino and S. R. White and S. Zhang, Phys. Rev. B 47, 7995 (1993).

${ }^{38}$ C. S. Hellberg and E. Manousakis, Phys. Rev. Lett. 78, 4609 (1997). 